\title{
Der Bock als guter Gärtner?
}

\author{
Nicht nur Staat und Verbraucherorganisationen, auch Unternehmen stellen \\ reglementierte Verbraucherinformationen bereit. Unternehmerische Verbrauche- \\ rinformationen beruhen auf gesetzlich vorgeschriebenen Informationspflichten \\ oder auf Informationsstandards, an die Unternehmen sich freiwillig binden. \\ Warum sollten Unternehmen - im eigenen und im gesellschaftlichen Interesse - \\ solche Informationen bereitstellen? Wo liegen die Grenzen der freiwilligen Ver- \\ braucherinformation durch Unternehmen?
}

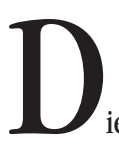

Von Ulf Schrader, Ingo Schoenheit und Ursula Hansen

on der Verbraucher ist eine zentrale Aufgabe der Verbraucherpolitik. Besondere Bedeutung kommt der Verbraucherinformation durch das Leitbild des nachhaltigen Konsums zu: Um nachhaltig konsumieren zu können, benötigen Menschen Informationen über die ökologischen und sozialen Merkmale von Produkten und Dienstleistungen. Bei diesen Merkmalen handelt es sich meist um so genannte Vertrauenseigenschaften, die Konsumenten weder vor dem Kauf durch Inspektion, noch während des Konsums durch eigene Erfahrung beurteilen können. Sie sind auf glaubwürdige Verbraucherinformationen angewiesen.

Traditionell werden als Akteure der Verbraucherinformation die Verbraucherorganisationen und staatliche Stellen betrachtet, die die Einhaltung grundlegender finanzieller und gesundheitlicher Verbraucherinteressen überwachen. Diese Akteurssicht folgt dem Verständnis einer ergänzenden und korrigierenden Verbraucherinformation, die als ,Gegengift" gegen unternehmerische Intransparenz wirkt. Gegenüber der Kommunikationspolitik von Herstellern und Händlern besteht aus dieser Perspektive der Grundverdacht, dass sie durch wirtschaftliche Interessen gefärbt und damit tendenziell unausgewogen und potenziell irreführend ist. Diese Sichtweise vernachlässigt aber, dass Unternehmen durch staatliche oder freiwillige „Informationsauflagenpolitik“ auch zur Beseitigung der Intransparenz beitragen können.

\section{Formen und Instrumente}

Selbstverständlich sind nicht alle von Unternehmen kommunizierten Informationen für Konsumenten auch Verbraucherinformationen. Ver- braucherinformation müssen für den Verbraucher nützlich sein und ihre Darbietung nach bestimmten transparenten Regeln erfolgen, die die sonstige Beliebigkeit der Informationsdarbietung eindeutig begrenzt (1). Klassische Instrumente der Unternehmenskommunikation, wie Werbung, Verkaufsförderung und Public Relations, sind keine Verbraucherinformation, sondern dem Bereich der sonstigen konsumrelevanten Informationen zuzuordnen (vgl. Abbildung 1).

Verbraucherinformationen der Anbieter beruhen entweder auf gesetzlichen Vorgaben oder auf freiwilliger Selbstbindung und liegen damit in standardisierter und vergleichbarer Form vor. Zur Verbraucherinformation nach gesetzlichen Vorgaben gehören etwa Preisauszeichnungen oder Inhaltskennzeichnungen. Auf Grundlage freiwilliger Selbstbindungen verwenden Unternehmen zum
Beispiel Sicherheitszeichen oder Gütesiegel wie das Bio-Siegel für Lebensmittel oder den Blauen Engel. Um überbetriebliche Vergleichbarkeit und Glaubwürdigkeit sicherzustellen, erfolgt die freiwillige Selbstbindung im Normalfall in Kooperation mit staatlichen und/oder zivilgesellschaftlichen Akteuren, häufig unter Beteiligung von Unternehmensverbänden. Insgesamt hängt das Ausmaß anbieterseitiger Verbraucherinformationen von den gesetzlichen Regelungen und der unternehmerischen Bereitschaft zu freiwilliger Selbstbindung ab. Auf beiden Wegen können Instrumente, die heute in den Bereich der ungeregelten, sonstigen konsumrelevanten Informationen fallen, zu Verbraucherinformationen gemacht werden. Denkbar ist dies beispielsweise für den wachsenden und stark ausdifferenzierten Bereich der Nachhaltigkeitsberichterstattung von Unternehmen.

Anbieter kommunizieren aber nicht nur direkt mit Konsumenten, sondern liefern zudem Input für die Verbraucherinformation staatlicher und zivilgesellschaftlicher Akteure. Warnungen und Auskünfte von Behörden basieren oft auf - freiwilligen oder verpflichtenden - Meldungen von Unternehmen. Auch Verbraucherorganisationen und mit ihnen kooperierende Forschungsinstitutionen nutzen beispielsweise für die Durchführung sozial-ökologischer Unternehmenstests unter anderem freiwillige Auskünfte von Unternehmen, die dann miteinander verglichen werden. (2).

Als privatwirtschaftliche Anbieter von Verbraucherinformationen agieren nicht nur Konsumgü-

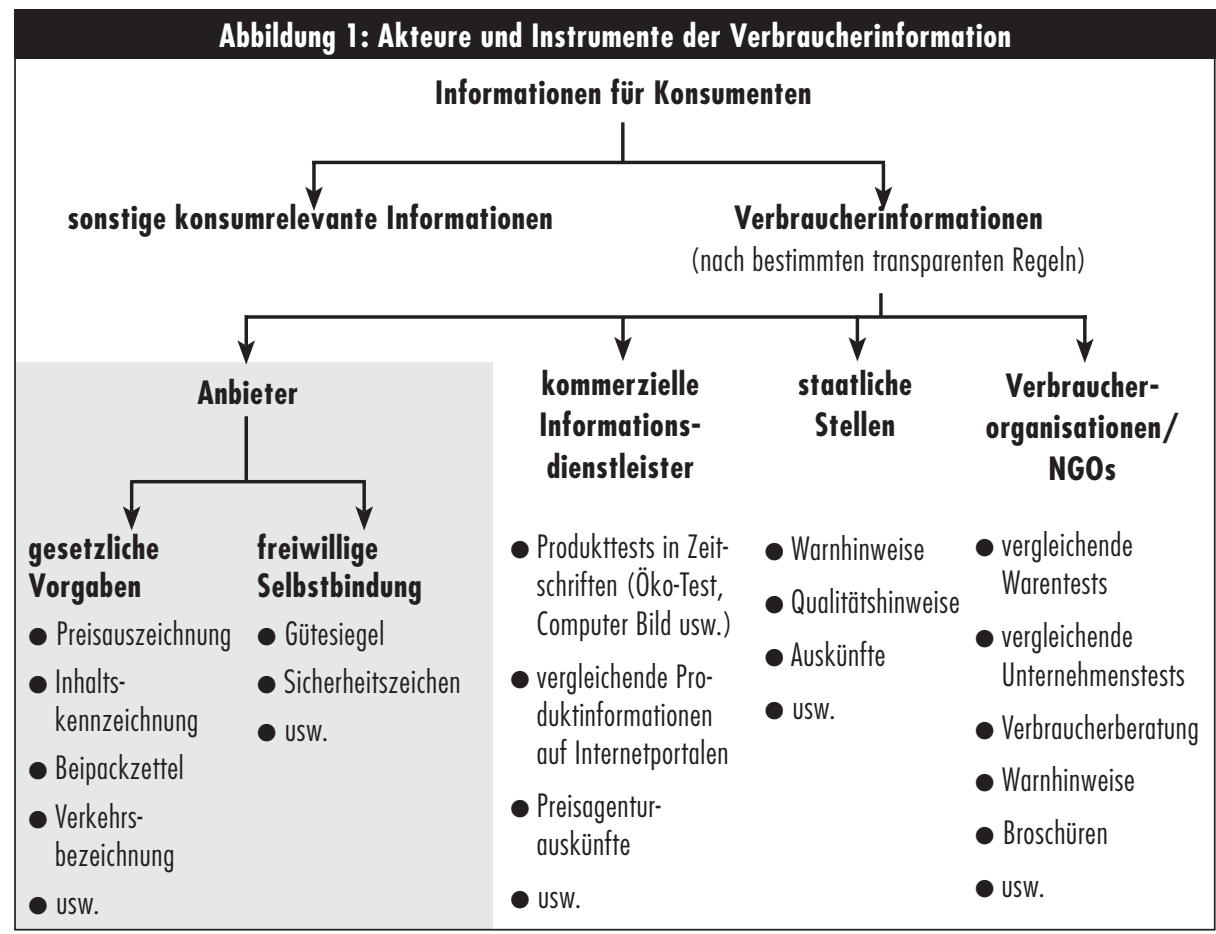

Quelle: Schoenheit 2003, unveröff. Manuskrip 
teranbieter, sondern auch Dienstleister, deren Geschäftstätigkeit im Verkauf von nützlichen Informationen für Konsumenten liegt. Diese Anbieter publizieren beispielsweise Publikumszeitschriften mit vergleichenden Produkt- und Dienstleistungstests oder sie betreiben Internetportale und Preisagenturen, die anbieterunabhängige vergleichende Informationen bereitstellen. Auch wenn Objektivität und Anbieterunabhängigkeit dieser Informationsangebote vor dem Hintergrund bestehender kommerzieller Interessen und Verflechtungen im Einzelfall kritisch zu überprüfen sind, stellen diese Akteure ebenfalls ein wichtiges Segment der Verbraucherinformation dar.

\section{- Gründe für Markt und Gesellschaft}

Die gesellschaftspolitische Forderung nach verstärkter Verantwortungsübernahme von Unternehmen für die Beseitigung der Intransparenz auf Konsumgütermärkten lässt sich inhaltlich und ökonomisch begründen, wobei beide Aspekte eng zusammenhängen. Die Bereitstellung zusätzlicher Verbraucherinformationen, etwa zu Inhaltsstoffen oder zu Produktionsbedingungen von Produkten, können andere Akteure allein zum Teil nur zu sehr hohen Kosten, zum Teil auch gar nicht leisten. Volkswirtschaftlich ist es zudem am effizientesten, Informationen dort bereitzustellen, wo dies die geringsten Kosten verursacht - in der Regel bei den Unternehmen selbst. Außerdem entspräche eine derartige Verantwortungszuschreibung auch dem Verursacherprinzip.

Wie abwegig es wäre, die Transparenzschaffung primär von Verbraucherorganisationen zu erwarten, zeigt schon ein Blick auf deren Budget: Die Stiftung Warentest, die Verbraucherzentralen der Länder und der Verbraucherzentralen Bundesverband (VZBV) verfügten 2001 über ein Budget von knapp 110 Millionen Euro (davon fast die Hälfte Eigeneinnahmen). Im selben Zeitraum betrug allein der Werbeetat der Unternehmen in Deutschland etwa 31,5 Milliarden Euro - also fast das 300-fache des Gesamtbudgets der Verbraucherorganisationen.

\section{- Gründe für Unternehmen}

Grundsätzlich sind Unternehmen dann bereit, freiwillig ihre Aktivitäten im Bereich der geregelten Verbraucherinformationen zu verstärken, wenn sie dadurch ihre Zielerreichung verbessern, wenn sich die Information also als business case darstellen lässt. So erhoffen sich Unternehmen Wettbewerbsvorteile bei umwelt- beziehungsweise gesundheits- bewusst oder sozial verantwortlich handelnden Konsumenten, wenn sie Produkte mit entsprechenden Kennzeichnungen anbieten. Anders als immer weitergehende Differenzierungen eigentlich homogener Produkte durch Design, Image oder technische „Spielereien“ bieten zielgruppengerechte Zusatzinformationen eine Möglichkeit, sich wirklich von den Wettbewerbern abzuheben.

Für Unternehmen kann sich erweiterte Verbraucherinformation auch lohnen, wenn sie nur bei wenigen Konsumenten einen direkten positiven Einfluss auf das Kaufverhalten hat. Durch konsumentenorientierte Informationsoffenheit kann das Unternehmen beweisen, dass es sich als Corporate Citizen versteht und sich dem Urteil aller Mitbürger stellt (3). Kritischen Anspruchsgruppen, die ansonsten die Legitimation anzweifeln und die Reputation beeinträchtigen könnten, wird so Protestpotenzial genommen, was langfristig zentral ist, um die licence to operate des Unternehmens zu sichern.

In Bereichen ohne gesetzliche Regelungen und freiwillige Standards, sind Unternehmen ebenfalls als Corporate Citizens gefordert. Hier bietet sich die Chance, ordnungspolitische Mitverantwortung zu übernehmen und Rahmenbedingungen aktiv zu gestalten. Dabei ist - etwa durch Kooperation auf Branchenebene - ein möglichst großer Anwendungsbereich und eine Alleinstellung der gesetzten Standards sicherzustellen, da nur so bei Konsumenten Glaubwürdigkeit erreicht und Informationsüberlastung vermieden werden kann.

\section{- Grenzen der Freiwilligkeit}

Die berechtigte Hoffnung auf eine positivere Rolle von Unternehmen bei der freiwilligen Bereitstellung von Verbraucherinformationen darf jedoch nicht den Blick auf die Grenzen dieses Ansatzes verstellen. Auch wenn Unternehmen ihre Kundenorientierung betonen - sie sind keine Verbraucherorganisationen und können auch keine sein. Letztlich werden sie Konsumenten nur in dem Maße informieren, in dem sie sich einen Nutzen davon versprechen. Daraus ergeben sich begrenzende Faktoren der freiwilligen Verbraucherinformation: Grenzen der Objektivität: Unternehmen sind begrenzt bereit, über negative Neben- und Folgewirkungen ihres Handelns genauso zu informieren wie über positive. Das Eingestehen kleinerer Versäumnisse dient zwar der Glaubwürdigkeit, grundlegende Selbstkritik ist jedoch kaum mit dem erhofften Wettbewerbsvorteil vereinbar.
Grenzen der Kostenübernabmebereitschaft: Glaubwürdige Verbraucherinformation kostet Geld. Begrenzte Informationen hat ihren Grund auch darin, dass Unternehmen viele Konsequenzen ihrer Wertschöpfung selbst nicht einschätzen können. Produktions- und Handelsverflechtungen in der globalisierten Wirtschaft sind oft extrem komplex; sie für die Konsumenten „gläsern“ zu machen, erfordert einen enormen und stetigen Aufwand. In Bereichen ohne transparente Selbstverpflichtungen entstehen zudem Kosten für die Verhandlung neuer Standards. Dabei besteht ein Trittbrettfahrerproblem, denn auch Unternehmen können von Standards profitieren, die sich nicht an ihrer Aushandlung beteiligt haben.

Grenzen der Offenlegung von „Betriebsgeheimnissen": Viele Unternehmen verbinden mit gläsernen Wertschöpfungsketten auch die Angst vor dem Verlust des eigenen Wissensvorsprungs. Verbraucherinformation ist auch Konkurrenten zugänglich - und wenn vollständige Transparenz über Inhaltsstoffe, Zulieferketten und Produktionsverfahren bestünde, könnte dies Wettbewerbsvorteile gefährden und langfristig auch Innovationsanreize reduzieren.

Grenzen der Glaubwürdigkeit: Die zuvor genannten Grenzen sind Konsumenten oft bekannt und begründen eine entsprechende Skepsis gegenüber vorhandenen freiwilligen unternehmerischen Verbraucherinformationen. Ihr besonderer Status als klar reglementierte Information ist vielen Konsumenten nicht deutlich, weil sie die hinter den Angaben stehenden Selbstverpflichtungen nicht kennen oder diesen nicht vertrauen. Sich die entsprechenden Informationen zu beschaffen, überfordert viele ohnehin schon unter Informationsüberlastung leidende Konsumenten.

\section{Die Autorlnnen}

Ingo Schoenheit ist geschäftsführender Vorstand des imug - Institut für Markt-Umwelt-Gesellschaft an der Universität Hannover e.V.

Kontakt: Tel. 0511/ 91115-20,

E-Mail: schoenheit@imug.de

Prof. Dr. Dr. h.c. Ursula Hansen leitet den Lehrstuhl Marketing I: Markt und Konsum an der Universität Hannover, Dr. Ulf Schrader ist wissenschaftlicher Mitarbeiter und Habilitand am 0.g. Lehrstuhl.

Kontakt: Tel. 0511/762-5629, E-Mail: us@muk.ifb.uni-hannover.de 


\section{- Politische Konsequenzen}

Noch ist unklar, unter welchen Bedingungen und inwieweit sich eine weitergehende freiwillige Verbraucherinformation insbesondere über soziale und ökologische Aspekte für die Anbieter lohnt. Hier sind verstärkte Forschungsanstrengungen im Hinblick auf die Wirkungen bei Konsumenten und Unternehmen gefragt, die eine entsprechende staatliche Forschungsförderung erfordern.

Generell ist der aktivierende Staat als Initiator und Motivator für branchenbezogene Verhandlungslösungen gefordert, an denen neben Unternehmen und ihren Verbänden auch Verbrau- cherorganisationen zu beteiligen sind. Ein solcher Ansatz verhandlungsorientierter Verbraucherinformationspolitik wird in Zukunft nicht immer, aber vielfach - wirklichkeitsnähere und erfolgreichere Lösungen hervorbringen, als dies eine gesetzlich verordnete Informationsauflagenpolitik allein erreichen kann (4)

\section{Anmerkungen}

(1) Vgl. Schoenheit, I. : Entwicklungsperspektiven der Verbraucherinformation und Verbraucherberatung. In: Mielenhausen, E. (Hrsg.): Verbraucherpolitik - Politik für den Verbraucher?, Osnabrück 1986, S. 97-127.

(2) Vgl. imug (Hrsg.): Unternehmenstest - Neue Heraus- forderungen für das Management der sozialen und ökologischen Verantwortung, München 1997.

(3) Vgl. zur Bedeutung der Kommunikation für das Corporate Citizenship Weiß, R.: Unternehmensführung in der Reflexiven Modernisierung: Global Corporate Citizenship, Gesellschaftsstrategie und Unternehmenskommunikation, Marburg 2002

(4) Vgl. Scharpf, F.W.: Lobbyismus für Verbraucherinteressen in der Verhandlungsdemokratie. In: Verbraucherzentrale Bundesverband e.V. (Hrsg.): Verbraucherschutz im globalen Markt, Berlin 2002, S. 8-16.

Hansen, U.: Wieviel Staat braucht die Verbraucherpolitik? In: FU Berlin (Hrsg.): Reden zur Verleihung der Ehrendoktorwürde an Frau Prof. Dr. U. Hansen, Berlin 2001.

\section{Der Verbraucher zwischen Unmündigkeit und politischer Manövriermasse}

Von Thilo Bode

BSE-verseuchtes Rindfleisch bescherte Deutschland zwar ein ganzes Verbraucherministerium samt grüner Ministerin, eine fortschrittliche verbraucherpolitische Gesamtkonzeption ist das Amt aber bis dato schuldig geblieben. Auf europäischer Ebene wird Verbraucherschutz, inzwischen sogar per EG-Vertrag, weniger als Schutz des Individuums vor den Kräften des Marktes verstanden, sondern vielmehr als Korrektiv und Voraussetzung für einen funktionierenden Binnenmarkt. Die Idee vom Verbraucher als aktiver Gestalter seines Marktes basiert auf dem Leitbild des ,,mündigen“ Verbrauchers: Einem Verbraucher, der informiert ist und verantwortlich handelt. In Berlin ist man noch nicht ganz so weit. Auf dem Papier hat RotGrün dieses Leitbild übernommen. In der Praxis muss das zuständige Ministerium jedoch die Interessen von Verbrauchern und Produzenten zugleich vertreten. Die Doppellaufgabe erklärt schon teilweise, warum das Bild dieser Regierung von mündigen Verbrauchern so unscharf bleibt. Verbraucherinteressen entsprechen eben nicht unmittelbar denen von Öko- und konventionellen Landwirten, von klein- und mittelständischer Ernährungswirtschaft, Lebensmittelindustrie und -handel.

Wer aber ist dieser Verbraucher? Wie liegen seine Interessen? Was macht ihn zu einem mündigen oder sogar ,guten“ Verbraucher? Die Entscheidung für Ökofleisch aus Paraguay und gegen konventionelle Schnitzel aus Schleswig-Holstein? Entmündigt sich der Supermarktkunde schon deshalb, weil er nicht im Bioladen kauft? Die während der Berliner „Grünen Woche“ aufgeflammte Diskussion um Verbraucher, Discounter und Agrarwende hat gezeigt, dass in Deutschland das
Leitbild des mündigen Verbrauchers häufig nur als politische Manövriermasse im Spiel ist. Die stockende Agrarwende - Schuld der Verbraucher. Die Verdrängung des Einzelhandels und die unbefriedigende Qualität vieler Nahrungsmittel - Verantwortung der Verbraucher.

Eine Politik, die Verbrauchern lediglich ihre eigenen Verfehlungen anlastet, aber ansonsten unmündig hält (wie zum Beispiel in der Frage der Höhe der Acrylamidbelastung einzelner Produkte), stärkt aber weder Verbraucher noch ihre Rechte, sondern verschaukelt sie. Wer von Verbrauchern Pflichten einfordern will, sollte erst einmal für die entsprechenden Rechte sorgen. Was ist die viel beschworene Qualität deutscher Lebensmittel denn wert, wenn man sie vor kritischen Nachfragen schützen muss?

Von der Opposition ist in diesem Punkt bedauerlicherweise auch nichts zu erhoffen. Und dies obwohl selbst konservative Ökonomen nicht mehr bestreiten, dass die Stärkung von Verbraucherrechten den Markt nicht schwächt sondern entwickelt und belebt.

\section{- Aktionen statt Appelle}

Statt Verbraucher mit moralischen Appellen zu traktieren, sollte die Regierung zunächst eine Kräftebalance zwischen Herstellern, Handel und Verbrauchern herstellen. Doch für ein Gleichgewicht braucht es Veränderung: Die Preise müssen etwa die tatsächlichen Kosten der Produktion widerspiegeln, inklusive aller Umweltbelastungen. Ökologisch hergestellte Produkte haben erst dann eine faire Chance im Wettbewerb, wenn die konventionelle Landwirtschaft gemäß dem Verursacherprinzip endlich für die von ihr verursachten Schäden wie Trinkwasserverschmutzung oder
Landschaftszerstörung auch gerade steht. Die Agrarpolitik fördert aber nach wie vor Einfalt statt Vielfalt, produziert Masse statt Klasse und leistet dem Verdrängungswettbewerb auf der Handelsebene weiteren Vorschub.

Auch wenn die Verbraucher sich möglicherweise nicht gemäß den Wünschen einer Verbraucherministerin verhalten, so ist ihr Handeln deswegen längst nicht verwerflich oder unmündig. Sie verhalten sich schlicht und einfach rational: Solange sich Verbraucher fürchten müssen, nur er oder sie allein zahlt für verantwortungsvollen Konsum und nicht alle anderen auch, wird er oder sie trotz guter Absichten weiter mit mehr oder weniger schlechtem Gewissen zum Schnäppchen greifen. Andere Annahmen sind so realistisch wie die Hoffnung, Mercedes würde freiwillig auf die Produktion von Solarmobilen umsteigen.

Die Politik sollte sich ihrer Verantwortung nicht durch die Bastelei neuer Verbraucherbilder entledigen, sondern für gewünschte Verhaltensänderungen Marktanreize schaffen: Volle Markttransparenz für die Verbraucher und eine Änderung der Produktionsbedingungen in der gesamten Land- und Ernährungswirtschaft - und damit letztlich kostendeckende Preise. Das kommt der Produktqualität zugute, nimmt die Verbraucher ernst und ist das gute Recht mündiger Bürger.

\section{Der Autor}

Thilo Bode ist Geschäftsführer der Verbraucherschutzorganisation Foodwatch.

Kontakt: foodwatch e.V., Brunnenstr. 181, 10119 Berlin, Tel. 030-28093-995 
(c) 20I0 Authors; licensee IÖW and oekom verlag. This is an article distributed under the terms of the Creative Commons Attribution Non-Commercial No Derivates License (http://creativecommons.org/licenses/by-nc-nd/3.o/), which permits unrestricted use, distribution, and reproduction in any medium, provided the original work is properly cited. 